\title{
Acute kidney injury as an independent risk factor for unplanned 90-day hospital readmissions
}

Simon Sawhney ${ }^{1,2,3^{*}}$, Angharad Marks ${ }^{1,2,3}$, Nick Fluck ${ }^{2}$, David J. McLernon ${ }^{1}$, Gordon J. Prescott ${ }^{1}$ and Corri Black ${ }^{1,2,3}$

\begin{abstract}
Background: Reducing readmissions is an international priority in healthcare. Acute kidney injury (AKI) is common, serious and also a global concern. This analysis evaluates AKI as a candidate risk factor for unplanned readmissions and determines the reasons for readmissions.

Methods: GLOMMS-II is a large population cohort from one health authority in Scotland, combining hospital episode data and complete serial biochemistry results through data-linkage. 16453 people (2623 with AKI and 13830 without AKI) from GLOMMS-II who survived an index hospital admission in 2003 were used to identify the causes of and predict readmissions. The main outcome was "unplanned readmission or death" within 90 days of discharge. In a secondary analysis, the outcome was limited to readmissions with acute pulmonary oedema. 26 candidate predictors during the index admission included AKI (defined and staged 1-3 using an automated e-alert algorithm), prior AKI episodes, baseline kidney function, index admission circumstances and comorbidities. Prediction models were developed and assessed using multivariable logistic regression (stepwise variable selection), C statistics, bootstrap validation and decision curve analysis.
\end{abstract}

Results: Three thousand sixty-five (18.6\%) patients had the main outcome (2702 readmitted, 363 died without readmission). The outcome was strongly predicted by AKI. Multivariable odds ratios for AKI stage 3; 2 and 1 (vs no AKI) were 2.80 (2.22-3.53); 2.23 (1.85-2.68) and 1.50 (1.33-1.70). Acute pulmonary oedema was the reason for readmission in $26.6 \%$ with $\mathrm{AKI}$ and $\mathrm{eGFR}<60$; and $4.0 \%$ with no AKI and eGFR $\geq 60$. The best stepwise model from all candidate predictors had a C statistic of 0.698 for the main outcome. In a secondary analysis, a model for readmission with acute pulmonary oedema had a C statistic of 0.853 . In decision curve analysis, AKI improved clinical utility when added to any model, although the incremental benefit was small when predicting the main outcome.

Conclusions: AKI is a strong, consistent and independent risk factor for unplanned readmissions - particularly readmissions with acute pulmonary oedema. Pre-emptive planning at discharge should be considered to minimise avoidable readmissions in this high risk group.

Keywords: Acute kidney injury, Acute renal failure, Patient readmission, Heart failure, Patient discharge, Decision support techniques, Prediction model, Clinical decision-making, Epidemiology, Prognosis

\footnotetext{
* Correspondence: simon.sawhney@abdn.ac.uk

${ }^{1}$ University of Aberdeen, Institute of Applied Health Sciences, Aberdeen, UK

${ }^{2} \mathrm{NHS}$ Grampian, Aberdeen, UK

Full list of author information is available at the end of the article
} International License (http://creativecommons.org/licenses/by/4.0/), which permits unrestricted use, distribution, and reproduction in any medium, provided you give appropriate credit to the original author(s) and the source, provide a link to the Creative Commons license, and indicate if changes were made. The Creative Commons Public Domain Dedication waiver (http://creativecommons.org/publicdomain/zero/1.0/) applies to the data made available in this article, unless otherwise stated. 


\section{Background}

Reducing unplanned readmissions after hospital discharge is an international priority for modern healthcare systems [1-3]. Readmission rates are driven by a mixture of health and social factors and some are potentially avoidable $[4,5]$. Globally, performance indicators, financial penalties, safety initiatives and prediction tools have been developed to reduce unplanned readmissions $[1,2]$. Clinical prediction tools combine available patient characteristics to predict a diagnostic or prognostic outcome [6]. Tools that predict whether patients leaving hospital are at a high risk of unplanned readmission would be helpful for the delivery of safe patient-centred care. However, current tools are limited by the inclusion of risk factors that are not widely accessible in routine clinical practice [7-9] (e.g., critical care scores or subjective social assessments). These limitations preclude a more general use of risk prediction tools for unplanned readmissions in clinical practice.

Acute kidney injury (AKI) is an abrupt change in kidney function, usually measured by a rising serum creatinine. As AKI is common across all hospital settings ( 1 in 7 hospital admissions), serious [10], and objectively measurable in a standardised fashion (using automated e-alerts) [11], it is a promising novel candidate risk factor for readmission. Good practice in AKI frequently requires rehydration with fluids and temporary discontinuation of cardiac medications until a patient improves [12]. People with AKI transition through multiple care providers and therefore good communication and awareness is needed to ensure that avoidable complications (e.g., overload, cardiovascular complications) do not occur. For this reason, AKI is now also the target of quality initiatives, including efforts to improve handovers at hospital discharge [13].

Previous work suggests an association between hospital AKI and increased hospital readmissions, but with methodological limitations. In one U.S study, the authors were unable to distinguish between planned and unplanned admissions. Pre-hospital creatinine values were also unavailable, which meant that only a minority subset of AKI (those who deteriorated during admission) could be analysed and AKI severity could not be staged [14]. A second U.S. study also associated AKI with readmissions, but was limited to survivors of AKI occurring in intensive care [15]. A third recently reported study from Canada showed a 1.5 -fold increase in 30 day readmissions among those with AKI in a propensitymatched cohort, but only a subset comprising those with more severe AKI recorded using ICD-10 codes were represented [16].

In this analysis, we evaluated the clinical utility of hospital AKI of all severities as a candidate risk factor for predicting and reducing unplanned hospital readmissions. We assessed whether AKI was an independent risk factor that could be used to guide decisions either in isolation or as part of a parsimonious clinical risk prediction tool. We also assessed whether the reasons for readmission were different for those with and without AKI, which would motivate the consideration of preemptive care plans at hospital discharge after AKI.

\section{Methods \\ Population}

This study includes all patients from the Grampian Laboratory Outcomes Morbidity and Mortality Study-II (GLOMMS-II) who were admitted to hospital in 2003 and survived to discharge $(n=16453)$. GLOMMS-II is a population cohort linking national and regional data sources for a single UK health authority (1999-2009). It includes routine hospital administrative data and the complete serial renal biochemistry profile for each patient [17-20]. Crucially for renal disease cohorts, all biochemistry is provided by a single biochemistry service, regardless of clinical location (inpatient, outpatient, community). This minimises any loss of baseline and follow up data and avoids selection biases in patient recruitment [20, 21]. Linkage to hospital episode data and the Scottish Renal Registry (SRR) provided mortality, admission episodes, morbidity events and chronic renal replacement therapy (RRT). Patients receiving chronic RRT prior to index hospital admission were excluded. The study had Regional Ethics Committee approval (14/NW/1371). Data were hosted and managed by Grampian Data Safe Haven [22].

\section{Outcomes - Unplanned readmission or death within 90 days}

The main outcome of interest was unplanned readmission or death within 90 days of discharge. We chose 90 days, because current international AKI guidelines recommend a reassessment at 3 months after AKI for the evaluation of future risk [23]. A distinction between unplanned and planned admissions is possible in Scotland because elective and emergency hospital episodes are specifically distinguished in the Scottish Morbidity Record (SMR01) by trained coders [24]. As 1.1\% died within 90 days without first being readmitted, this more severe endpoint was combined with readmission for the logistic regression. As a sensitivity analysis, we also analysed readmission using a multinomial approach (i.e., readmission vs alive and not readmitted; and death without readmission vs alive and not readmitted), which yielded similar results. As additional sensitivity analyses, we also generated models for the main outcome at 30 and 60 days. Finally, because we identified a substantial increase in readmissions due to acute pulmonary oedema (a potentially modifiable reason) among those with AKI, we conducted a secondary analysis of the outcome restricted to readmission with acute pulmonary oedema. 


\section{Follow up}

Follow up was from the date of discharge from the original (index) admission until the next emergency readmission or death. The index admission, as previously described, was the first admission with AKI, or last admission without AKI in 2003 [20]. 94.6\% of the study population had evidence of follow-up (e.g., linkage to blood tests) up to or beyond the end of the study. Of the remainder, as migration out of Grampian was negligible for the period and age-mix of the cohort [25], those without follow-up beyond the end of the study were assumed to be alive without achieving the main outcome.

\section{Covariates and candidate predictors}

Based on previous studies [7, 9, 14], a combination of renal measures, comorbidities, social measures and admission circumstances were included as candidate predictors. Renal measurements included AKI severity (stages $0-3,0$ being no AKI) for an index episode in 2003, the presence of AKI episodes in the prior 91-1095 days (i.e., AKI in the 3 years prior to the baseline lookback period), baseline estimated glomerular filtration rate (eGFR) and the presence of a $>20 \%$ worsening of serum creatinine from baseline to hospital discharge (i.e., non-recovery). AKI and baseline kidney function were determined using the "Kidney Disease: Improving Global Outcomes" (KDIGO) criteria [23, 26]. We used a KDIGO-based AKI e-alert definition to identify all discrete AKI episodes lasting up to 90 days from 2000 until the end of 2003 [20]. A summary of this AKI definition is also provided in Table 1 with more detail available elsewhere [20]. AKI severity was the highest stage achieved (1-3) within each AKI episode period with respect to the baseline identified at the point of identification of each new AKI episode. The rolling lookback period ensured that the baseline creatinine was updated between AKI episodes so that further rises in creatinine after an AKI episode could be distinguished either as recurrent AKI (further rapid rises above a prior AKI episode and meeting KDIGO cirteria) or CKD progression/ non-recovery (elevated creatinine following a prior AKI

Table $1 \mathrm{KDIGO}$-based acute kidney injury criteria for this study (as described in [20])

\begin{tabular}{ll}
\hline AKI criteria & Definition \\
\hline Index AKI episode (lasting & $\begin{array}{l}\text { Serum creatinine } \geq 1.5 \text { times higher than } \\
\text { the median of all creatinine values } 8-90 \\
\text { days ago; or } 91-365 \text { days ago if no tests } \\
\text { between } 8 \text { and } 90 \text { days; } \\
\text { or serum creatinine } \geq 1.5 \text { times higher } \\
\text { than the lowest creatinine within } 7 \text { days; } \\
\text { or serum creatinine }>26 \mu \mathrm{mol} / \mathrm{L} \text { higher } \\
\text { than the lowest creatinine within } 48 \mathrm{~h}\end{array}$ \\
& $\begin{array}{l}\text { Any episode occurring } 91-1095 \text { days } \\
\text { prior to index episode }\end{array}$ \\
\hline
\end{tabular}

episode but no actual acute rise meeting KDIGO cirteria). Baseline eGFR was reported using the Chronic Kidney Disease Epidemiology Collaboration (CKD-EPI) creatinine equation [27].

Comorbidities were the "international classification of diseases" (ICD-10) codes for Charlson comorbidities from the 5 years prior to admission as previously described and validated [28]. Social and demographic measures included age, sex, residential care (long-term care home or skilled nursing home), deprivation and rural home location as previously described [20, 29]. Metrics of admission circumstances were the number of hospital admissions in the past year, length of hospital stay for the index admission, emergency or elective admission, and admission to a medical ward or intensive care. For the causes of readmission, ICD-10 diagnoses for the readmission episode were also recorded. Based on previous work and validation studies these were acute coronary syndrome (with or without infarction) (I21-I22, I20) [30, 31]; cerebrovascular disease (G45, I60-67) [32-34]; lower respiratory tract infection (pneumonia or bronchitis) (J10-18, J20-22) [35, 36]; and acute pulmonary oedema in the context of heart failure (I50) $[37,38]$.

\section{Statistical analyses}

We plotted Kaplan-Meier curves for readmission-free survival with risk tables showing numbers alive, readmitted and dead up to 1 year after hospital discharge.

To compare the reasons for hospital readmission in those with and without AKI, we recorded the readmission ICD-10 codes for readmission diagnoses. Based on prior research [14], and the recognised role of fluids and cardiac medications in AKI [12], we reported four specific diagnoses: acute coronary syndrome, cerebrovascular event, lower respiratory tract infection, acute pulmonary oedema.

We performed univariable and multivariable logistic regression to assess the association of each candidate predictor with 90 day readmission or death. We performed multivariable logistic regression using a full model containing all candidate predictors. To determine a "best stepwise model", we then used all candidate predictors with stepwise backwards elimination of predictors with a $p$-value $\geq 0.01[6,39,40]$. This $p$-value threshold was chosen to approximate to the Bayesian Information Criterion (BIC) for the large sample size of the analysis $[41,42]$. We also developed models using the same stepwise procedure but limiting candidate predictors to administrative data only (including age); age and renal biochemistry only; age alone; and AKI alone. Based on prior knowledge and graphical inspection, we modelled age and eGFR continuously using linear and quadratic terms [43]. We repeated this modelling procedure for an additional outcome limited to readmissions with acute pulmonary oedema, and for outcomes at 30 and 60 days. 


\section{Assessment of model performance}

We assessed model performance by testing discrimination and calibration. Discrimination measures how well a model distinguishes between those with and without an outcome. We calculated the area under the receiver operating characteristic curve (AUC), which can be considered equivalent to a C-statistic [44]. The value of a $\mathrm{C}$ statistic lies between 0.5 and 1; 0.5 meaning that the model is no better than a coin toss at discrimination and 1 meaning perfect discrimination. Pairwise comparison of $\mathrm{C}$ statistics for different models was performed as previously outlined elsewhere $[45,46]$. Calibration is a measure of how well the predicted probabilities of an outcome from a model agree with the observed probabilities of the outcomes. We developed a calibration plot of mean observed probability vs mean predicted probability of outcomes within tenths of increasing predicted risk. All points lying on a calibration slope of 1 indicates perfect agreement, and a slope of less than 1 indicates over-fitting of the model [6]. We also performed a Hosmer-Lemeshow goodness-of-fit test to assess for a statistically significant difference between observed and predicted values, standardised for sample size using a method described elsewhere [47].

Validation of a model using the same data as used to develop the model (known as apparent validation) usually results in optimistic measures of performance. Therefore, we performed internal validation of the best stepwise model using bootstrap resampling. We generated 500 bootstrapped datasets with replacement from the original dataset. For each bootstrapped sample we applied the same backward selection modelling process used to derive the original best stepwise model. The $\mathrm{C}$ statistic was calculated for each of the 500 bootstrapped models both in the bootstrap data set and the original data set. The difference between the two $\mathrm{C}$ statistics for each sample was found and averaged over the 500 samples. This average difference indicated the optimism of the $\mathrm{C}$ statistic in the original model and is an estimate of internal validity. This procedure enabled us to provide a $\mathrm{C}$ statistic and calibration slope corrected for model optimism [6]. As stepwise procedures can lead to instability in variable selection we also recorded the variable inclusion frequencies for each of the bootstrap models using the backwards elimination procedure with a $\mathrm{p} \geq 0.01$ threshold $[42,48]$.

\section{Model application}

Even if a risk factor improves model discrimination and calibration, a model may not result in better decisions. Decision curve analysis is a recent method of assessing the clinical usefulness of different models at an appropriate threshold for clinical use [49]. We used decision curve analysis to compare each model and also strategies of "predict all" or "predict none".
Decision curve analysis is a plot of the "net benefit" against "threshold probabilities". Such a plot identifies the range of threshold probabilities for which the model is of clinical value i.e., can be used to guide decisions. The threshold probability indicates the cut-off for classifying a prediction as positive or negative for an outcome [50]. An acceptable threshold may differ for clinicians and patients making decisions in different clinical contexts. A threshold of close to zero would imply that false-positive predictions are acceptable to ensure that no patients are missed. A higher threshold would involve targeting only higher risk patients with fewer falsepositives. Net benefit measures the trade-off between true-positives and false-positives in a prediction model at different threshold probabilities. It is a sum of true-positive minus false-positive predictions weighted by the threshold probability as described in the equation below [49].

$$
\begin{aligned}
\text { Net benefit }= & \left(\frac{\text { true positive }}{\text { total sample size }}\right)-\left[\left(\frac{\text { false positive }}{\text { total sample size }}\right)\right. \\
& \left.\times\left(\frac{\text { threshold probability }}{1-\text { threshold probability }}\right)\right]
\end{aligned}
$$

The model with the highest net benefit at a given threshold, has the greatest clinical value. At a threshold probability of zero a policy of targeting all patients would be of greatest value as there would be no penalty from false-positives. At higher thresholds, an alternative approach guided by a prediction model may provide greater benefit. In this study, if a clinician wished to identify patients with higher than average risk of readmission, this would correspond to a threshold probability of $0.2-0.4$ and a prediction model would need to show greater net benefit over this pre-specified range.

Decision curve analysis comprehensively compares models across all thresholds, however we also calculated the integrated discrimination improvement (IDI) and categorical net reclassification improvement (NRI) with categories of low, medium and high risk using a thresholds of 0.1 and 0.3 for the main outcome and 0.01 and 0.1 for the outcome limited to readmissions with pulmonary oedema for those familiar with these alternative metrics [51, 52].

Statistical analysis was performed in Stata SE version 13 using "dca", "incrisk" and "roccomp" packages for the assessments of model performance [45, 53-55]. We also developed a web-based application to illustrate how risk predictions changed in the presence of AKI. This was performed in R using the package "shiny" and "personograph"[56-58].

\section{Results}

\section{Cohort characteristics}

Cohort formation and characteristics of 16453 patients surviving an index hospital admission (2623 with AKI 
and 13830 without AKI), with and without readmission or death by 90 days, are described in Fig. 1 and Table 2 . Univariable odds ratios are provided in the final column of Table 2. Three thousand sixty five of 16453 patients (18.6\%) were readmitted or died within 90 days of hospital discharge (including 363 deaths occurring without readmission and 269 readmissions with pulmonary oedema). Those with readmission or death were older, more frequently in residential care, had more previous hospital admissions, longer hospital stay at index admission and more comorbidities. They also experienced more AKI during index admission, more prior AKI episodes, and had worse kidney function at baseline and at discharge.

\section{Reasons for readmission after AKI}

Reasons for readmission grouped by AKI and baseline eGFR are summarised in Fig. 2. There was little difference in readmissions due to cerebrovascular episodes, and a modest increase in readmissions due to lower respiratory tract infection or acute coronary episodes among those with eGFR $<60 \mathrm{ml} / \mathrm{min} / 1.73 \mathrm{~m}^{2}$. However, there was a substantial increase in readmissions due to acute pulmonary oedema in those with AKI and eGFR $<60 \mathrm{ml} / \mathrm{min} /$ $1.73 \mathrm{~m}^{2}$ (respectively $26.6 \% ; 13.1 \% ; 7.0$ and $4.0 \%$ for AKI and eGFR < 60; no AKI and eGFR < 60; AKI and eGFR $\geq$ 60; no AKI and eGFR $\geq 60 \mathrm{ml} / \mathrm{min} / 1.73 \mathrm{~m}^{2}$ ). This trend was the same whether acute pulmonary oedema was used in any diagnostic position (as above) or restricted to the main diagnosis.

\section{Relationship between AKI and unplanned readmission or death}

Figure 1 describes the status of patients over the first 90 days after discharge from index admission. There were 16453 patients who survived an index hospital admission. Patients with AKI (vs no AKI) and baseline eGFR $<60$ (vs eGFR $\geq 60 \mathrm{ml} / \mathrm{min} / 1.73 \mathrm{~m}^{2}$ ) had poorer outcomes, with greater occurrence of unplanned readmission or death. Figure 3 is a Kaplan-Meier plot of readmission-free survival stratified by AKI and baseline eGFR. At all time-points up to 1 year, patients with AKI had poorer outcomes than those without AKI and patients with baseline eGFR $<60$ had poorer outcomes than those with baseline eGFR $\geq 60 \mathrm{ml} / \mathrm{min} / 1.73 \mathrm{~m}^{2}$.

\section{AKI as an independent predictor of unplanned readmission or death}

Table 3 summarises multivariable logistic regression using all candidate predictors (full model), and using a stepwise regression (best stepwise model). AKI (staged $0-3$ ) independently predicted readmission. Four comorbidities (cancer, cardiac failure, diabetes and chronic pulmonary disease) were also present in the best model. AKI was one of the most consistently selected variables, present in $100 \%$ of 500 bootstrapped models. Age,

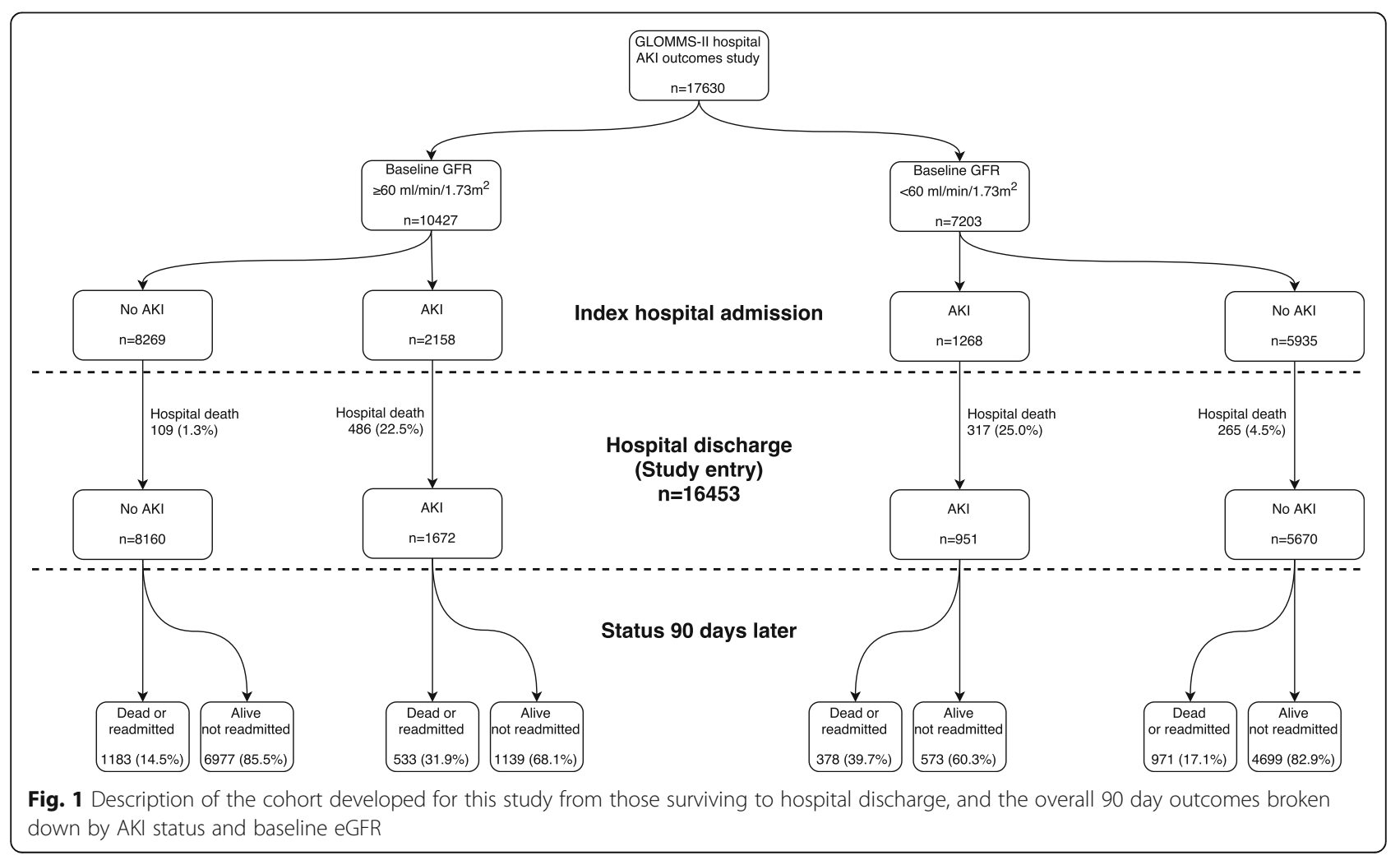


Table 2 Cohort characteristics

\begin{tabular}{|c|c|c|c|c|c|c|}
\hline \multirow{2}{*}{$\bar{N}$} & \multicolumn{2}{|c|}{$\begin{array}{l}90 \text { day readmission } \\
\text { or death (\%) }\end{array}$} & \multicolumn{2}{|c|}{$\begin{array}{l}90 \text { day readmission free survival } \\
\text { (\%) baseline impairment }\end{array}$} & \multicolumn{2}{|c|}{ Univariable odds ratio $(95 \% \mathrm{Cl})$} \\
\hline & 3065 & & 13388 & & & \\
\hline Age (median, IQR) & 74 & $(62-83)$ & 69 & $(55-79)$ & 1.20 (/10 years) & $(1.17-1.23)$ \\
\hline Male sex & 1372 & $(44.8)$ & 5770 & $(43.1)(0)$ & 1.07 & $(0.99-1.16)$ \\
\hline Residential care & 277 & $(9.0)$ & 471 & $(3.5)$ & 2.72 & $(2.34-3.18)$ \\
\hline Deprived (highest quintile) & 298 & $(9.7)$ & 1088 & (8.1) & 1.22 & $(1.06-1.39)$ \\
\hline Rural (settlement <3000) & 735 & $(24.0)$ & 3720 & $(27.8)$ & 0.82 & $(0.75-0.90)$ \\
\hline \multicolumn{7}{|l|}{ Admission context } \\
\hline No admissions past year ${ }^{a}$ & 1896 & $(61.9)$ & 10461 & $(78.1)$ & 1.38 (/admission) & $(1.34-1.43)$ \\
\hline 1 admission past year & 593 & $(19.3)$ & 1872 & $(14.0)$ & & \\
\hline 2 admissions past year & 244 & (8.0) & 620 & $(4.6)$ & & \\
\hline $3+$ admissions past year & 332 & $(10.8)$ & 435 & $(3.2)$ & & \\
\hline Length of stay (median, IQR) & 7 & $(2-16)$ & 3 & $(1-9)$ & 1.05 (/7 days) & $(1.04-1.06)$ \\
\hline Emergency admission & 2326 & $(75.9)$ & 7760 & $(58.0)$ & 2.28 & $(2.09-2.50)$ \\
\hline Medical ward admission & 1889 & (61.6) & 6570 & $(49.1)$ & 1.67 & $(1.54-1.81)$ \\
\hline Intensive care admission & 98 & $(3.2)$ & 366 & $(2.7)$ & 1.18 & $(0.94-1.47)$ \\
\hline \multicolumn{7}{|l|}{ Renal function } \\
\hline No AKI & 2154 & $(70.3)$ & 11676 & $(87.2)$ & (reference group) & \\
\hline AKI stage 1 & 528 & $(17.2)$ & 1190 & (8.9) & 2.41 & $(2.15-2.69)$ \\
\hline AKI stage 2 & 233 & (7.6) & 341 & $(2.5)$ & 3.70 & $(3.12-4.40)$ \\
\hline AKI stage 3 & 150 & $(4.9)$ & 181 & (1.4) & 4.49 & $(3.60-5.60)$ \\
\hline No prior AKI episodes ${ }^{a}$ & 2526 & (82.4) & 12188 & $(91.0)$ & 1.71 (/episode) & $(1.58-1.85)$ \\
\hline 1 prior AKI episode & 414 & $(13.5)$ & 1000 & $(7.5)$ & & \\
\hline 2+ prior AKI episodes & 125 & $(4.1)$ & 200 & $(1.5)$ & & \\
\hline Baseline eGFR (median, IQR) ${ }^{a}$ & 63 & $(48-83)$ & 66 & $(52-87)$ & $0.94\left(/ 10 \mathrm{ml} / \mathrm{min} / 1.73 \mathrm{~m}^{2}\right)$ & $(0.93-0.96)$ \\
\hline Baseline eGFR 0-29 & 213 & $(6.9)$ & 502 & (3.7) & & \\
\hline Baseline eGFR 30-44 & 423 & (13.8) & 1461 & $(10.9)$ & & \\
\hline Baseline eGFR 45-59 & 713 & $(23.3)$ & 3309 & $(24.7)$ & & \\
\hline Baseline eGFR $\geq 60$ & 1716 & $(56.0)$ & 8116 & $(60.6)$ & & \\
\hline Discharge creatinine $20 \%>$ baseline & 520 & $(17.0)$ & 1167 & $(8.7)$ & 2.14 & $(1.91-2.39)$ \\
\hline \multicolumn{7}{|l|}{ Comorbidity } \\
\hline Cancer & 410 & (13.4) & 973 & (7.3) & 1.97 & $(1.74-2.23)$ \\
\hline Cardiac failure & 317 & $(10.3)$ & 592 & (4.4) & 2.49 & $(2.16-2.88)$ \\
\hline Cerebrovascular disease & 231 & $(7.5)$ & 580 & $(4.3)$ & 1.80 & $(1.54-2.11)$ \\
\hline Dementia & 96 & (3.1) & 163 & $(1.2)$ & 2.62 & $(2.03-3.39)$ \\
\hline Diabetes & 336 & $(11.0)$ & 776 & $(5.8)$ & 2.00 & $(1.75-2.29)$ \\
\hline Hemiplegia & 28 & $(0.9)$ & 71 & $(0.5)$ & 1.73 & $(1.11-2.68)$ \\
\hline Liver disease & 59 & (1.9) & 156 & $(1.2)$ & 1.66 & $(1.23-2.25)$ \\
\hline Myocardial infarction & 257 & (8.4) & 638 & $(4.8)$ & 1.83 & $(1.57-2.13)$ \\
\hline Peptic ulcer disease & 81 & (2.6) & 278 & $(2.1)$ & 1.28 & $(1.00-1.64)$ \\
\hline Peripheral vascular disease & 162 & (5.3) & 452 & (3.4) & 1.60 & $(1.33-1.92)$ \\
\hline Pulmonary disease & 346 & $(11.3)$ & 704 & $(5.3)$ & 2.29 & $(2.00-2.62)$ \\
\hline Rheumatic disease & 82 & $(2.7)$ & 289 & $(2.2)$ & 1.25 & $(0.97-1.60)$ \\
\hline
\end{tabular}

Abbreviations: AKI acute kidney injury, $C l$ confidence interval, eGFR estimated glomerular filtration rate, IQR inter-quartile range a Modelled here linearly per $10 \mathrm{ml} / \mathrm{min} / 1.73 \mathrm{~m}^{2}$ increase and reported in categories for clarity 


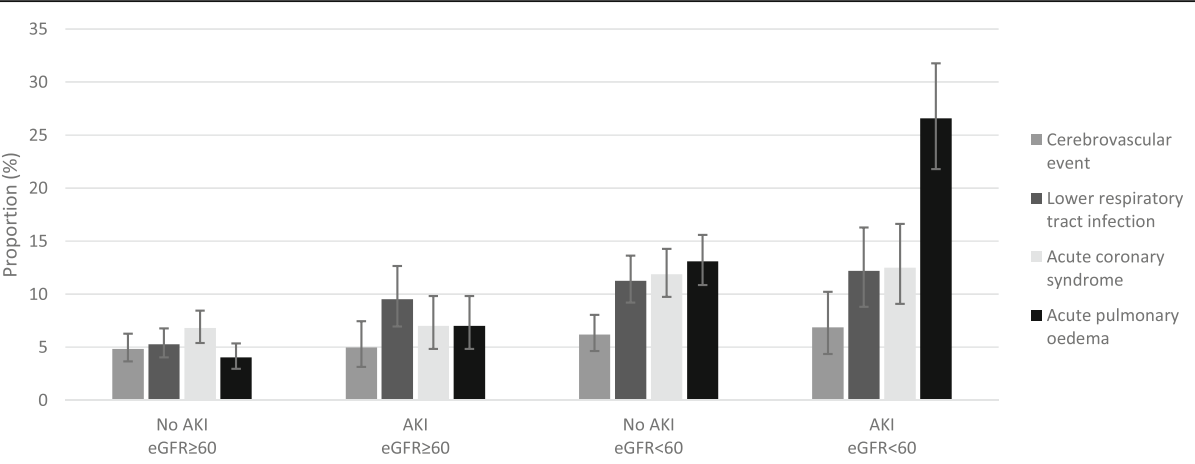

Fig. 2 Reasons for unplanned hospital readmission among those people in the cohort readmitted within 90 days of hospital discharge

residential care, number of previous admissions, emergency admission and cancer were also selected in 100\% of bootstrapped models. In sensitivity analysis, this did not change if those who died without readmission were not included in the outcome. Models for 60 and 30 day outcomes were also similar (Additional file 1: Table S1). When the outcome for analysis was restricted to readmissions with pulmonary oedema, age, AKI and history of previous cardiac failure were the most consistently selected variables (Additional file 1: Table S2).

\section{Performance of prediction models}

Table 4 reports the predictors that were significant in stepwise regression when the procedure was limited to groups of candidate predictors. Based on model discrimination (C statistic), performance of the best stepwise

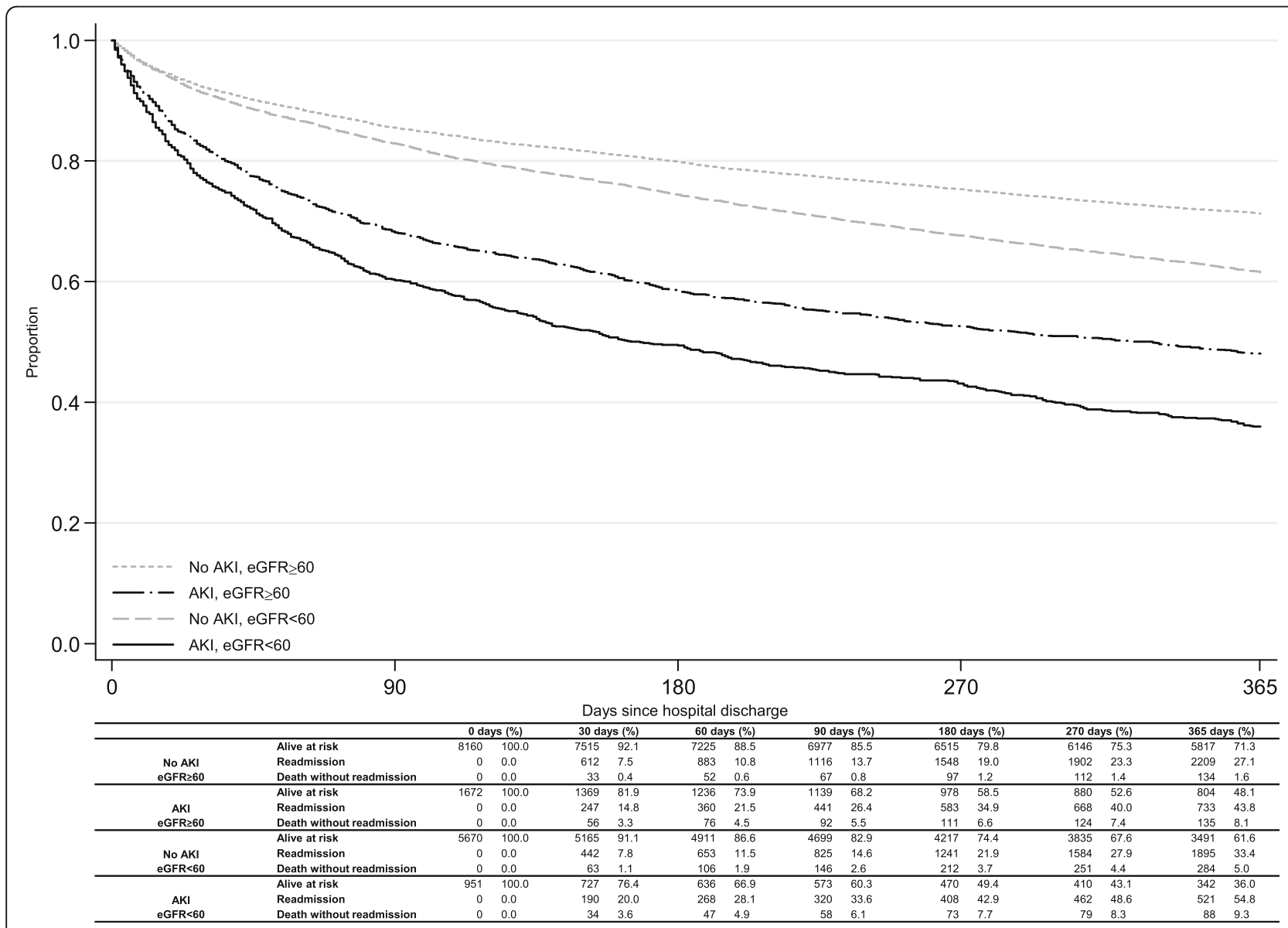

Fig. 3 Unadjusted curves of readmission-free survival with risk table of death and readmission by AKI status and baseline eGFR 
Table 3 Stepwise model of unplanned 90 day readmission or death after hospital discharge

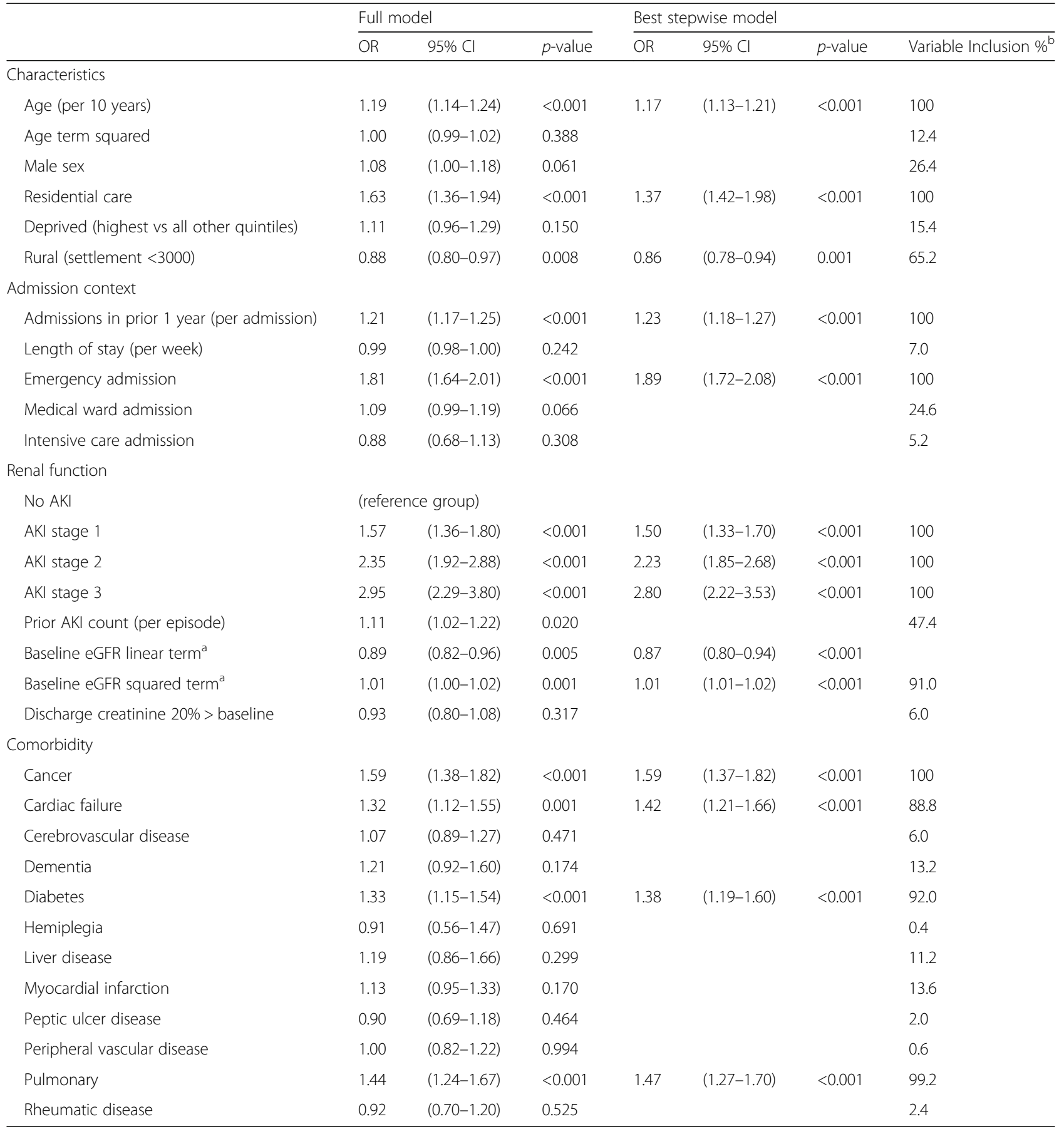

Abbreviations: $A K I$ acute kidney injury, $\mathrm{Cl}$ confidence interval, eGFR estimated glomerular filtration rate, OR odds ratio

${ }^{a}$ Modelled per $10 \mathrm{ml} / \mathrm{min} / 1.73 \mathrm{~m}^{2}$ increase with a combination of linear and quadratic terms. Variable inclusion \% applies to the baseline eGFR variable overall ${ }^{b}$ In 500 bootstrapped datasets

model (0.698) was no different to a full model containing all predictors (0.699), and showed statistically significant incremental improvement over models limited to administrative data only; renal biochemistry and age; age alone; and AKI alone. The C statistic for the best stepwise model (0.698) was 0.695 after bootstrap correction for optimism. The bootstrap calibration slope was 0.97 (0.89-1.06), showing excellent agreement at all but the very highest levels of predicted risk on a calibration plot (Additional file 2: Figure S1). For the model limited to readmissions with acute pulmonary oedema, the $\mathrm{C}$ statistic was substantially larger $(0.853 ; 0.845$ after bootstrap 
Table 4 Comparison of prediction models and model discrimination

\begin{tabular}{|c|c|c|c|c|c|c|}
\hline & Full model & $\begin{array}{l}\text { Best stepwise } \\
\text { model }\end{array}$ & $\begin{array}{l}\text { Administrative } \\
\text { data only model }\end{array}$ & $\begin{array}{l}\text { Biochemistry } \\
+ \text { age model }\end{array}$ & Age alone model & AKI alone \\
\hline \multicolumn{7}{|l|}{ Characteristics } \\
\hline Age & * & * & * & * & * & \\
\hline Age term quadratic term & * & & & * & * & \\
\hline Male sex & * & & & & & \\
\hline Residential care & * & * & * & & & \\
\hline Deprived (highest quintile) & * & & & & & \\
\hline Rural (settlement <3000) & * & * & * & & & \\
\hline \multicolumn{7}{|l|}{ Admission context } \\
\hline Admissions in prior 1 year (per admission) & * & * & * & & & \\
\hline Length of stay (per week) & * & & & & & \\
\hline Emergency admission & * & * & * & & & \\
\hline Medical ward admission & * & & * & & & \\
\hline Intensive care admission & * & & & & & \\
\hline \multicolumn{7}{|l|}{ Renal function } \\
\hline AKI stages $0-3$ & * & * & & * & & * \\
\hline Prior AKI count (per episode) & * & & & * & & \\
\hline Baseline eGFR (linear and quadratic) & * & * & & * & & \\
\hline Discharge creatinine $20 \%$ > baseline & * & & & & & \\
\hline \multicolumn{7}{|l|}{ Comorbidity } \\
\hline Cancer & * & * & * & & & \\
\hline Cardiac failure & * & * & * & & & \\
\hline Cerebrovascular disease & * & & & & & \\
\hline Dementia & * & & & & & \\
\hline Diabetes & * & * & * & & & \\
\hline Hemiplegia & * & & & & & \\
\hline Liver disease & $*$ & & & & & \\
\hline Myocardial infarction & * & & & & & \\
\hline Peptic ulcer disease & $*$ & & & & & \\
\hline Peripheral vascular disease & * & & & & & \\
\hline Pulmonary & * & * & * & & & \\
\hline Rheumatic disease & * & & & & & \\
\hline Model C statistic & 0.699 & 0.698 & 0.685 & 0.655 & 0.594 & 0.587 \\
\hline 95\% confidence interval & $(0.688-0.709)$ & $(0.688-0.709)$ & $(0.675-0.696)$ & $(0.644-0.666)$ & $(0.582-0.605)$ & $(0.578-0.596)$ \\
\hline $\begin{array}{l}P \text {-value for AUC comparison with the next } \\
\text { most complex model }\end{array}$ & - & 0.536 & $<0.001$ & $<0.001$ & $<0.001$ & 0.344 \\
\hline
\end{tabular}

Abbreviations: AKI acute kidney injury, eGFR estimated glomerular filtration rate

correction for optimism), with more substantial incremental improvements over other models (Additional file 1: Table S3). Again, the bootstrap calibration slope was not significantly different from $1(0.90,0.70-1.14)$. Further details of the calibration of both models is provided in Additional file 2: Figure S1, Additional file 3: Figure S2, Additional file 4: Figure S3 and Additional file 5: Figure S4.

\section{Model application}

Figure 4 shows decision curve analysis plots contrasting the net benefit of the different prediction models for the main outcome of death or readmission within 90 days (4A) and for the secondary analysis of readmission with acute pulmonary oedema (4B). At the a priori specified threshold of 0.2-0.4 for the main analysis, all models performed better than predicting readmission in all or 


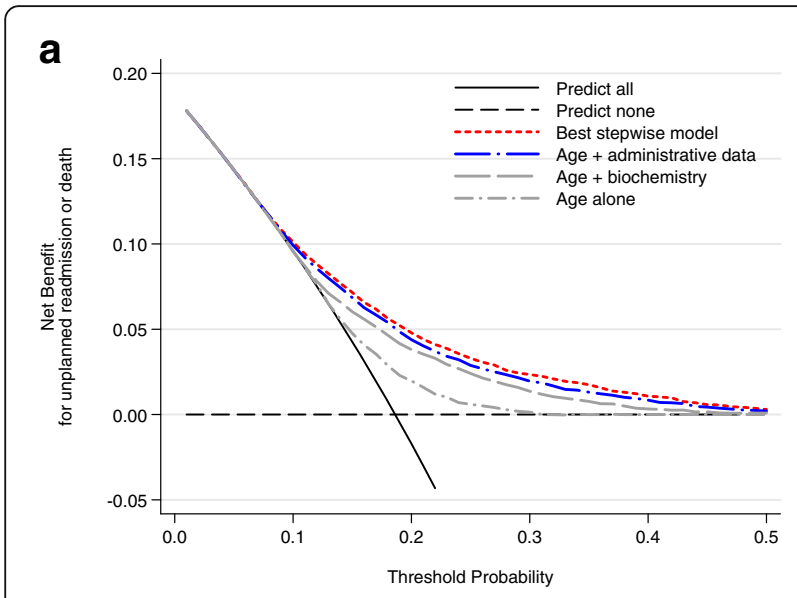

b

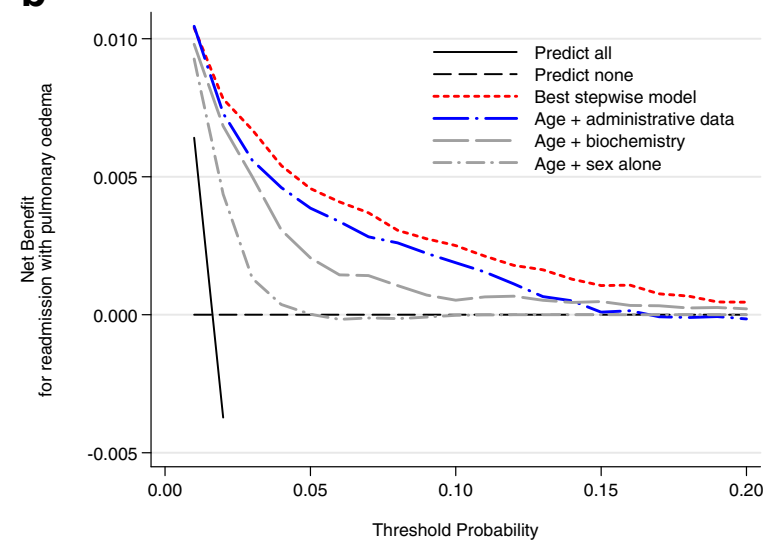

Fig. 4 Decision curve analysis comparing the net benefit of prediction models for readmission or death 90 days after discharge (a) or for readmission with acute pulmonary oedema 90 days after discharge (b)

no patients. Age provided modest net benefit, with small incremental improvement from adding biochemistry or administrative data to the model and best performance from the best stepwise model from all candidate predictors. For the acute pulmonary oedema model the best stepwise model was again superior to all other models, with larger incremental improvement across all thresholds.

For the main analysis best stepwise model (vs administrative data) the categorical $\left.\mathrm{NRI}_{[0.1,}, 0.3\right]$ was $+4.6 \%(+2.7$ to +7.0$)$ (event $\mathrm{NRI}_{[0.1,0.3]}+2.0 \%[+0.6$ to +3.7$]$; nonevent $\left.\mathrm{NRI}_{[0.1,} 0.3\right]+2.7 \%[+0.6$ to +4.2$\left.]\right)$ and IDI was $+0.012(+0.009$ to +0.017$)$ with positive values indicating overall improvement in the prediction probabilities. For the acute pulmonary oedema best stepwise model (vs administrative data) the categorical $\left.\mathrm{NRI}_{[0.01,} 0.1\right]$ was $+11.8 \%(+0.1$ to +19.6$)\left(\right.$ event $\mathrm{NRI}_{[0.01,0.1]}+1.5 \%[-1.9$ to +5.4$]$; non-event $\mathrm{NRI}_{[0.01,0.1]}+10.3 \%[+0.0$ to +16.3$\left.]\right)$ and IDI was $+0.009(+0.001$ to +0.024$)$.

Additional file 6: Figure S5 illustrates how predictions change in the presence of AKI, and the accessibility of the data required to generate predictions. This calculator is available on request by contacting the authors. Using the best stepwise model, a 70 year old man with diabetes who is admitted urgently with severe AKI requiring dialysis would have a predicted 90 day risk of readmission or death of $42 \%$. The same man without AKI would have a predicted risk of $21 \%$. In contrast, using the administrative data model (which includes no measure of AKI) the predicted risk would be the same for those with and without AKI (26\%).

\section{Discussion}

AKI is common, objectively assessable using serial serum biochemistry (or e-alerts), and associated with increased mortality. Our analysis shows that AKI is also a strong independent predictor of 90 day unplanned readmission or death. Furthermore, there were striking differences in the cause of readmissions between those with and without AKI. Up to 1 in 4 readmissions after AKI were related to acute pulmonary oedema - a potentially modifiable reason for readmission.

Despite being a strong predictor, the incremental improvement in overall predictions after adding AKI to the best alternative model was small, both based on decision curve analysis and on categorical NRI. This is often the case in saturated prediction models [59], but may suggest limited added value of combining renal biochemistry and hospital episode data for general population risk predictions. Nevertheless, our study shows that AKI is still an attractive risk factor in clinical practice because it is common, strongly associated with readmission, and associated with a complication (pulmonary oedema) that could be targeted as part of a pre-emptive discharge plan. This is reinforced by our secondary analysis, which showed that when the outcome was limited to readmissions with acute pulmonary oedema, the absolute performance of the model (C statistic 0.853 ) and the incremental improvement with the addition of AKI were both greater.

Our analysis is consistent with and extends previous research. Previous work in the U.S. and Canada has associated AKI with readmission, but only studied either a subset of AKI that could be identified using only inpatient biochemistry data without distinguishing between planned and unplanned readmissions [14], or the subset of AKI treated in intensive care [15], or the subset of AKI recognised in ICD-10 coding [16]. We extend previous work using a U.K. cohort by capturing all hospital AKI using all biochemistry (inpatient and outpatient), by focusing on unplanned admissions, by using AKI severity and prior AKI episodes as novel predictors and by assessing the incremental benefit of AKI in risk prediction. The bootstrapped $C$ statistic presented in our study (0.695) is consistent with previous UK-based studies predicting 12 month (apparent C statistic 0.685) [8] and 30 day (bootstrapped $C$ statistic 0.699) [9] readmissions. 
The strongest predictors (age, admission circumstances, cancer, cardiac failure, diabetes and pulmonary disease) also agree with those consistently reported in the literature [7].

Strengths of this analysis include the use of a large unselected population with complete biochemistry and administrative data capture, minimising misclassification due to missing baseline renal data. The linkage of serial renal biochemistry to administrative data demonstrates AKI as a novel and objective predictor that could be reproduced in future research and updated with each admission in real-time clinical practice. The role of AKI and baseline eGFR in readmissions with acute pulmonary oedema was particularly striking. We note previous work has identified heart failure as a common reason for 30 day hospital readmissions [16], and shown that among patients with heart failure, those who develop AKI have more readmissions [60]. This analysis provides the complementary finding that among all hospitalised patients, those with AKI have substantially more emergency readmissions due to acute pulmonary oedema - a potentially preventable reason for readmission [5]. Collectively, these results provide a motivation for improving handovers and medication plans when discharging patients with AKI [13], and for an AKI follow-up clinic [61]. A particular population to focus on could be those with a history of CKD or heart failure, and interventions to evaluate in this group could include volume reassessments, diuretic algorithms and medication reconciliation.

In this study we demonstrated that the use of serial renal laboratory measurements can lead to incremental improvements in clinical risk prediction models. While not the focus of this study, we recognise that a number of other repeatedly measured laboratory parameters (such as discharge sodium, albumin and C-reactive protein) may lead to further incremental improvements. These were not available in this analysis, nor used in previous UK-based readmission prediction tools, and would be an appropriate next step for future research $[8,9]$. We also recognise that the role of AKI in our health care system in Scotland may not be generalisable to other regions and health care systems. This may also be the case for other predictors that may differ for health care systems in other countries (e.g., residential care and intensive care). Additional validation would be valuable, because any variation in other regions would also improve our understanding of the circumstances that lead to poorer outcomes after AKI. ICD-10 coding is also subject to variation and misclassification. While sensitive for the clinical diagnosis of heart failure, previous work has shown that the specificity of ICD-10 coding of heart failure can vary depending on whether only the main diagnosis or all diagnoses are included [37, 38]. Nevertheless, we found the trend was the same in both situations. Similarly, because our cohort was originally constructed to observe long-term outcomes in those with kidney disease, the cohort inception was in 2003. We analysed from the cohort inception date because analysis of any later period would have introduced a survivorship bias. While our objective biochemical AKI criteria will not be affected by the cohort dates, recent initiatives to improve the recognition and care of AKI may alter the rates of early readmission. This would be important to reassess, but would be unlikely to materially change the message of our study. Finally, AKI is a clinical diagnosis incorporating the clinical context changes in serum creatinine and urine output. Our identification of AKI involved an algorithm for changes in creatinine in routine data. A problem common to all AKI studies involving large populations is the potential for misclassification bias when blood testing is sometimes infrequent and context is not available to ensure AKI and CKD are classified appropriately.

\section{Conclusions}

Overall, this study indicates that AKI is a strong predictor of unplanned readmissions. Acute pulmonary oedema is a potential driver of the increased readmissions in AKI patients. This raises the possibility that some readmissions after AKI may be avoidable by careful pre-emptive planning after AKI to prevent the development of pulmonary oedema.

\section{Additional files}

Additional file 1: Table S1. Comparison of prediction models and model discrimination for different time points. Table $\mathbf{S} 2$ Stepwise model of unplanned 90 day readmission with pulmonary oedema after hospital discharge. Table S3 Comparison of prediction models and model discrimination for death or readmission with pulmonary oedema. (DOCX $33 \mathrm{~kb})$

Additional file 2: Figure S1. Calibration plot for best stepwise prediction model of 90 day readmission or death. Circles represent observed and predicted readmission in deciles of predicted risk. Histograms represent the distributions of patients with (top) and without (bottom) readmission. (PDF $31 \mathrm{~kb}$ )

Additional file 3: Figure S2. Calibration plot for best stepwise prediction model of 90 day readmission with acute pulmonary oedema. Circles represent observed and predicted readmission in deciles of predicted risk. Histograms represent the distributions of patients with (top) and without (bottom) readmission. (PDF $40 \mathrm{~kb}$ )

Additional file 4: Figure S3. Hosmer-Lemeshow test standardised for sample size for model of 90 day readmission or death with plots of quintiles of observed and predicted risk. (PDF $43 \mathrm{~kb}$ )

Additional file 5: Figure S4. Hosmer-Lemeshow test standardised for sample size for model of 90 day readmission with acute pulmonary oedema with plots of quintiles of observed and predicted risk. (PDF $43 \mathrm{~kb}$ )

Additional file 6: Figure S5. Illustration of clinical risk prediction model using examples with and without AKI. (PDF 647 kb)

\section{Abbreviations}

AKI: Acute kidney injury; AUC: Area under the receiver operating characteristic curve; BIC: Bayesian Information Criterion; Cl: Confidence interval; CKD: Chronic kidney disease; CKD-EPI: Chronic Kidney Disease Epidemiology Collaboration; eGFR: estimated glomerular filtration rate; GLOMMS: Grampian Laboratory Outcomes Morbidity and Mortality Study; 
ICD: International classification of diseases; IDI: Integrated discrimination improvement; IQR: Inter-quartile range; KDIGO: Kidney disease: improving global outcomes; NRI: Net reclassification improvement; OR: Odds ratio; RRT: Renal replacement therapy; SMR: Scottish morbidity record; SRR: Scottish renal registry; U.S.: United States

\section{Acknowledgements}

None.

\section{Funding}

We acknowledge the data management support of Grampian Data Safe Haven (DaSH) and the associated financial support of NHS Research Scotland, through NHS Grampian investment in the Grampian DaSH. SS is supported by a Clinical Research Training Fellowship from the Wellcome Trust (Ref 102729/Z/13/Z). We also acknowledge the support from The Farr Institute of Health Informatics Research. The Farr Institute is supported by a 10-funder consortium: Arthritis Research UK, the British Heart Foundation, Cancer Research UK, the Economic and Social Research Council, the Engineering and Physical Sciences Research Council, the Medical Research Council, the National Institute of Health Research, the National Institute for Social Care and Health Research (Welsh Assembly Government), the Chief Scientist Office (Scottish Government Health Directorates), the Wellcome Trust, (MRC Grant Nos: Scotland MR/K007017/1). These funders had no role in the study design, in the collection, analysis and interpretation of data, in the writing of the report, or in the decision to submit this article for publication.

\section{Availability of data and materials}

Participant level de-identified data used for this study are held by Grampian Data Safe Haven. These data are available provided the necessary permissions have been obtained. Depending on the nature of the request, this may include Caldicott guardian, NHS Grampian Research and Development, and local Research Ethics Committee approval. Further information is available at http://www.abdn.ac.uk/iahs/facilities/grampian-data-safe-haven.php and requests for data may be made to Dr Corri Black on behalf of Grampian Data Safe Haven, corri.black@abdn.ac.uk.

\section{Authors' contributions}

Research idea and study design: SS; data acquisition: AM, CB, SS; data analysis/ interpretation: AM, CB, DM, GP, NF, SS; statistical analysis: AM, DM, GP, SS; supervision or mentorship: AM, CB. Each author contributed important intellectual content during manuscript drafting or revision and accepts accountability for the overall work by ensuring that questions pertaining to the accuracy or integrity of any portion of the work are appropriately investigated and resolved. SS is the guarantor for the study. All authors had full access to all of the data (including statistical reports and tables) in the study and can take responsibility for the integrity of the data and the accuracy of the data analysis. All authors read and approved the final manuscript.

\section{Competing interests}

The authors declare that they have no competing interests.

\section{Consent for publication}

Not applicable.

\section{Ethics approval and consent to participate}

Approval was obtained from Privacy Advisory Committee (study number XRB14137) and the Regional Ethics Committee (reference 14/NW/1371) who waived the requirement for informed consent for this study.

\section{Author details}

${ }^{1}$ University of Aberdeen, Institute of Applied Health Sciences, Aberdeen, UK. ${ }^{2} \mathrm{NHS}$ Grampian, Aberdeen, UK. ${ }^{3}$ Farr Institute@Scotland, Aberdeen, UK.

Received: 5 November 2016 Accepted: 20 December 2016 Published online: 06 January 2017

\section{References}

1. Nolte $E$, Roland R, Guthrie S, Brereton L. Preventing emergency readmissions to hospital: a scoping review. Santa Monica: RAND Corporation; 2012. Available at: http://www.rand.org/pubs/technical_ reports/TR1198.html. Accessed 6 June 2016.
2. Atkins DKD. Reducing readmissions—destination or journey? JAMA Intern Med. 2016;176(4):493-5.

3. Organisation for Economic Co-operation and Development. OECD Health Ministerial Meeting. Health System Priorities in the Aftermath of the Crisis. 2010; Available at: https://www.oecd.org/health/ministerial/46098360.pdf. Accessed 6 June 2016

4. Parliamentary and Health Service Ombudsman. A report of investigations into unsafe discharge from hospital. 2016; Available at: http://www. ombudsman.org.uk/reports-and-consultations/reports/health/a-report-ofinvestigations-into-unsafe-discharge-from-hospital. Accessed 6 June 2016.

5. Wiebe N, Klarenbach SW, Allan GM, Manns BJ, Pelletier R, James MT Bello A, Hemmelgarn BR, Tonelli M. Potentially preventable hospitalization as a complication of CKD: a cohort study. Am J Kidney Dis. 2014;64(2):230-8.

6. Steyerberg EW. Clinical prediction models. 1st ed. New York: Springer; 2009.

7. Kansagara D, Englander H, Salanitro A, et al. Risk prediction models for hospital readmission: a systematic review. JAMA. 2011:306(15):1688-98.

8. Billings J, Dixon J, Mijanovich T, Wennberg D. Case finding for patients at risk of readmission to hospital: development of algorithm to identify high risk patients. Br Med J. 2006;333(7563):327.

9. Billings J, Blunt I, Steventon A, Georghiou T, Lewis G, Bardsley M. Development of a predictive model to identify inpatients at risk of readmission within 30 days of discharge (PARR-30). BMJ Open. 2012;2(4): e001667. doi:10.1136/bmjopen-2012-001667.

10. Chertow GM, Burdick E, Honour M, Bonventre JV, Bates DW. Acute kidney injury, mortality, length of stay, and costs in hospitalized patients. J Am Soc Nephrol. 2005;16(11):3365-70.

11. NHS England. Patient safety alert on standardising the early identification of Acute Kidney Injury. 2014; 2014: Available at: https://www.england.nhs.uk/ patientsafety/2014/06/09/psa-aki/. Accessed 6 June 2016.

12. National Institute for Health and Care Excellence: Prevention, detection and management of acute kidney injury up to the point of renal replacement therapy. NICE 2013, Guideline 169: London, UK.

13. NHS England. Commissioning for Quality and Innovation (CQUIN). Guidance for 2015/16. 2015. Available at: https://www.england.nhs.uk/wp-content/ uploads/2015/03/9-cquin-guid-2015-16.pdf. Accessed 6 June 2016.

14. Koulouridis I, Price LL, Madias NE, Jaber BL. Hospital-acquired acute kidney injury and hospital readmission: a cohort study. Am J Kidney Dis. 2015;65(2): 275-82.

15. Horkan CM, Purtle SW, Mendu ML, Moromizato T, Gibbons FK, Christopher KB. The association of acute kidney injury in the critically ill and postdischarge outcomes: a cohort study. Crit Care Med. 2015;43(2):354-64.

16. Silver SA, Harel Z, McArthur E, Nash DM, Acedillo R, Kitchlu A, Garg AX, Chertow GM, Bell CM, Wald R. 30-Day readmissions after an acute kidney injury hospitalization. Am J Med. (in press) http://dx.doi.org/10.1016/j. amjmed.2016.09.016

17. Marks A, Fluck N, Prescott GJ, Robertson L, Simpson WG, Cairns Smith W, Black C. Looking to the future: predicting renal replacement outcomes in a large community cohort with chronic kidney disease. Nephrol Dial Transplant. 2015;30(9):1507-17.

18. Sawhney S, Fluck N, Fraser SD, Marks A, Prescott GJ, Roderick PJ, Black C. KDIGO-based acute kidney injury criteria operate differently in hospitals and the community - findings from a large population cohort. Nephrol Dial Transplant. 2016;31(6):922-9.

19. Coresh J, Turin TC, Matsushita K, Sang Y, Ballew SH, Appel $\sqcup$, Arima H, Chadban SJ, Cirillo M, Djurdjev O, Green JA, Heine GH, Inker LA, Irie F, Ishani A, Ix JH, Kovesdy CP, Marks A, Ohkubo T, Shalev V, Shankar A, Wen CP, de Jong PE, Iseki K, Stengel B, Gansevoort RT, Levey AS. CKD prognosis consortium: decline in estimated glomerular filtration rate and subsequent risk of end-stage renal disease and mortality. JAMA. 2014; 311(24):2518-31.

20. Sawhney S, Marks A, Fluck N, Levin A, Prescott GJ, Black C. Intermediate and long-term outcomes of survivors of acute kidney injury episodes: a large population-based cohort study. Am J Kidney Dis. 2017;69(10):18-28.

21. Sawhney S, Fluck N, Marks A, Black B. Diagnostic sensitivity and false positive AKI alerts through unlinking of an integrated Grampian biochemistry service. 2016. Available online: http://dx.doi.org/10.13140/RG.2. 1.3279.4480. Accessed 6 June 2016

22. University of Aberdeen. Grampian Data Safe Haven. 2014; Available at http://www.abdn.ac.uk/iahs/facilities/grampian-data-safe-haven.php. Accessed 6 June 2016 
23. Kidney Disease: Improving Global Outcomes (KDIGO) Acute Kidney Injury Work Group. KDIGO clinical practice guideline for acute kidney injury. Kidney inter. 2012;2(Suppl):1-138.

24. ISD Scotland. Data sources and clinical coding. 2016. Available at: http:// www.isdscotland.org/Health-Topics/Hospital-Care/Beds/Data_Sources_and_ Clinical_Coding.pdf. Accessed 1 Nov 2016.

25. General Register Office for Scotland: Grampian migration report 2010. Available at: http://www.nrscotland.gov.uk/files/statistics/migration/ Migration-Reports/grampian-migration-report.pdf. Accessed 11 Nov 2016

26. Kidney Disease: Improving Global Outcomes (KDIGO) CKD Work Group. KDIGO clinical practice guideline for the evaluation and management of chronic kidney disease. Kidney inter. 2013;3(Suppl):1-150.

27. Levey AS, Stevens LA, Schmid CH, Zhang Y, Castro AF, Feldman HI, Kusek JW, Eggers P, Van Lente F, Greene T, Coresh J. A New equation to estimate glomerular filtration rate. Ann Intern Med. 2009:150(9):604-12.

28. Johnston MC, Marks A, Crilly MA, Prescott GJ, Robertson LM, Black C. Charlson index scores from administrative data and case-note review compared favourably in a renal disease cohort. Eur J Public Health. 2015; 25(3):391-6.

29. ISD Scotland. Deprivation and urban rural measurements in ISD. 2004 Available at: http://www.isdscotlandarchive.scot.nhs.uk/isd/files//Measuring_ deprivation_in_ISD_v3.pdf. Accessed 6 June 2016.

30. Australian Institute of Health and Welfare. Acute coronary syndrome: validation of the method used to monitor incidence in Australia. 2014 Available at: http://www.aihw.gov.au/WorkArea/DownloadAsset.aspx?id= 60129547560. Accessed 1 Oct 2016

31. Metcalfe A, Neudam A, Forde S, Liu M, Drosler S, Quan H, Jetté N. Case definitions for acute myocardial infarction in administrative databases and their impact on in-hospital mortality rates. Health Serv Res. 2013;48(1):290-318.

32. Kokotailo RA, Hill MD. Coding of stroke and stroke risk factors using international classification of diseases, revisions 9 and 10. Stroke. 2005;36(8):1776.

33. Ghia D, Thomas PR, Cordato DJ, Worthington JM, Cappelen-Smith C, Griffith N, Hanna I, Hodgkinson SJ, McDougall A, Beran RG. Validation of emergency and final diagnosis coding in transient ischemic attack: South Western Sydney transient ischemic attack study. Neuroepidemiology. 2010;35(1):53-8.

34. McCormick N, Bhole V, Lacaille D, Avina-Zubieta J. Validity of diagnostic codes for acute stroke in administrative databases: a systematic review. PLOS ONE. 2015;10(8):e0135834.

35. Skull S, Andrews R, Byrnes G, Campbell D, Nolan T, Brown G, Kelly H. ICD-10 codes are a valid tool for identification of pneumonia in hospitalized patients aged >65 years. Epidemiol Infect. 2007;136(2):232-40.

36. Henriksen DP, Nielsen SL, Laursen CB, Hallas J, Pedersen C, Lassen AT. How well Do discharge diagnoses identify hospitalised patients with communityacquired infections? A validation study. PLoS ONE. 2014;9(3):e92891.

37. Frolova N, Bakal JA, McAlister FA, Rowe BH, Quan H, Kaul P, Ezekowitz JA. Assessing the Use of International Classification of Diseases-10th Revision Codes From the Emergency Department for the Identification of Acute Heart Failure. JACC: Heart Failure. 2015;3(5):386-91.

38. Quan H, Li B, Saunders LD, Parsons GA, Nilsson Cl, Alibhai A, Ghali WA. IMECCHI investigators: assessing validity of ICD-9-CM and ICD-10 administrative data in recording clinical conditions in a unique dually coded database. Health Serv Res. 2008:43(4):1424-41.

39. Sauerbrei $W$. The use of resampling methods to simplify regression models in medical statistics. J R Stat Soc: Ser C: Appl Stat. 1999;48(3):313-29.

40. Sauerbrei $W$, Royston $P$, Binder $H$. Selection of important variables and determination of functional form for continuous predictors in multivariable model building. Stat Med. 2007;26(30):5512-28.

41. Teräsvirta T, Mellin I. Model selection criteria and model selection tests in regression models. Scand J Stat. 1986;13(3):159-71.

42. Royston P, Sauerbrei W. Multivariable model-building. 1st ed. Chichester: Wiley; 2008.

43. Shlipak MG, Matsushita K, Arnlov J, Inker LA, Katz R, Polkinghorne KR, Rothenbacher D, Sarnak MJ, Astor BC, Coresh J, Levey AS, Gansevoort RT. CKD Prognosis Consortium: Cystatin C versus creatinine in determining risk based on kidney function. N Engl J Med. 2013;369(10):932-43.

44. Austin PC, Steyerberg EW. Interpreting the concordance statistic of a logistic regression model: relation to the variance and odds ratio of a continuous explanatory variable. BMC Med Res Methodol. 2012;12(1):82.

45. Cleves MA. From the help desk: Comparing areas under receiver operating characteristic curves from two or more probit or logit models. Stata J. 2002; 2(3):301-13.
46. DeLong ER, DeLong DM, Clarke-Pearson DL. Comparing the areas under two or more correlated receiver operating characteristic curves: a nonparametric approach. Biometrics. 1988:44(3):837-45.

47. Paul P, Pennell ML, Lemeshow S. Standardizing the power of the HosmerLemeshow goodness of fit test in large data sets. Stat Med. 2013;32(1):67-80.

48. Sauerbrei W, Schumacher M. A bootstrap resampling procedure for model building: Application to the cox regression model. Stat Med. 1992;11(16): 2093-109.

49. Vickers AJ, Van Calster B, Steyerberg EW. Net benefit approaches to the evaluation of prediction models, molecular markers, and diagnostic tests. BMJ. 2016:352:i6.

50. Steyerberg EW, Vergouwe Y. Towards better clinical prediction models: seven steps for development and an ABCD for validation. Eur Heart J. 2014; 35(29):1925-31.

51. Pencina MJ, D'Agostino RB, D'Agostino RB, Vasan RS. Evaluating the added predictive ability of a new marker: from area under the ROC curve to reclassification and beyond. Stat Med. 2008;27(2):157-72.

52. Pencina MJ, Steyerberg EW, D'Agostino RB. Extensions of net reclassification improvement calculations to measure usefulness of new biomarkers. Stat Med. 2010:30(1):11-21.

53. StataCorp. Stata statistical software: release 13. College Station: StataCorp LP 2013.

54. Vickers AJ. Decision Curve Analysis. Available at: https://www.mskcc.org/ departments/epidemiology-biostatistics/health-outcomes/decision-curveanalysis-01. Accessed 1 Nov 2016.

55. Pepe M. Diagnostic and Biomarkers Statistical (DABS) Center. Risk Prediction Package. Available at: http://research.fhcrc.org/diagnostic-biomarkers-center/ en/software/ppsoft.html. Accessed 1 Nov 2016.

56. R Core Team. R: A language and environment for statistical computing. Vienna: R Foundation for Statistical Computing; 2016. https://www.R-project.org/. Accessed 20 Dec 2016.

57. Chang W, Cheng J, Allaire J, Xie Y, McPherson J. shiny: Web Application Framework for R. R package version 0.14. 2016 https://CRAN.R-project.org/ package=shiny. Accessed 20 Dec 2016.

58. Kuiper J, Marshall I. personograph: Pictographic Representation of Treatment Effects. R package version 0.1.3. 2015 https://CRAN.R-project.org/ package=personograph. Accessed 20 Dec 2016

59. Leening MJG, Vedder MM, Witteman JCM, Pencina MJ, Steyerberg EW. Net reclassification improvement: computation, interpretation, and controversies: a literature review and Clinician's guide. Ann Intern Med. 2014;160(2):122-31.

60. Thakar CV, Parikh PJ, Liu Y. Acute kidney injury (AKI) and risk of readmissions in patients with heart failure. Am J Cardiol. 2012;109(10):1482-6.

61. Silver SA, Goldstein SL, Harel Z, et al. Ambulatory care after acute kidney injury: an opportunity to improve patient outcomes. Can J Kidney Health Dis. 2015;2:36.

\section{Submit your next manuscript to BioMed Central and we will help you at every step:}

- We accept pre-submission inquiries

- Our selector tool helps you to find the most relevant journal

- We provide round the clock customer support

- Convenient online submission

- Thorough peer review

- Inclusion in PubMed and all major indexing services

- Maximum visibility for your research

Submit your manuscript at www.biomedcentral.com/submit
) Biomed Central 